\title{
A CLASS OF PRIMARY ABELIAN GROUPS CHARACTERIZED BY ITS SOCLES
}

\author{
PATRICK KEEF
}

(Communicated by Warren J. Wong)

\begin{abstract}
The $t$-product of a family $\left\{G_{i}\right\}_{i \in I}$ of abelian $p$-groups is the torsion subgroup of $\prod_{i \in I} G_{i}$, which we denote by $\prod_{i \in I}^{t} G_{i}$. The $t$-product is, in the homological sense, the direct product in the category of abelian $p$-groups. Let $\mathscr{R}^{s}$ be the smallest class containing the cyclic groups that is closed with respect to direct sums, summands, and $t$-products. It is proven that two groups in $\mathscr{R}^{s}$ are isomorphic iff their socles are isomorphic as valuated vector spaces. This generalizes a classical result on direct sums of torsion-complete groups. As is frequently the case with homomorphisms defined on products, the index sets will be assumed to be nonmeasurable.
\end{abstract}

In this note, by the term "group", we will mean an abelian $p$-group, where $p$ is some fixed prime. The notation and terminology will follow [F1]. We also assume some rudimentary knowledge of valuated vector spaces (e.g., [F2]).

The height function on a group $G$ induces a valuation on the socle $G[p]=$ $\{g \in G: p g=0\}$. A frequently repeated theme in abelian group theory involves the relationship between the structure of $G$ as a group and $G[p]$ as a valuated vector space. In particular, if $G$ and $H$ are groups whose socles are isometric (i.e., isomorphic as valuated vector spaces), when can we conclude that $G \cong H$ ? This question is of particular importance in the case of separable groups (i.e., those with no nonzero elements of infinite height). If $G$ is torsion-complete or a direct sum of cyclics, then $G \cong H$ whenever $G[p]$ and $H[p]$ are isometric. However, much work has been done to show that this is almost never true if $G$ fails to be of this type (e.g., [HM, Du, Cu, MS, S]).

To get more useful results, one has to restrict the class being considered; the class of all groups is simply too large. The question then becomes: find classes $C$ such that whenever, $G, H$ are in $C, G$ is isomorphic to $H$ iff $G[p]$ is isometric to $H[p]$. If this is the case, we will say that the groups in $C$ are characterized by their socles. There are two main results of this type in the literature. In [FI] it was shown that the $p^{\omega+1}$-projectives are characterized by their socles ( $G$ is the $p^{\omega+1}$-projective iff there is a subgroup $P \subseteq G[p]$ with $G / P$ a direct sum of cyclics). Of more relevance for this work, in [H1] it was

Received by the editors December 10, 1990.

1980 Mathematics Subject Classification (1985 Revision). Primary 20K10, $20 \mathrm{~K} 40$.

Key words and phrases. Abelian p-group, direct product, direct sum.

This research was supported by NSF (RUI) grant DMS-9000129.

Presented to the AMS at its meeting at UC Irvine, October 1990. 
shown that the direct sums of torsion-complete groups are characterized by their socles. It is this result that we generalize.

The $t$-product $\left\{G_{i}\right\}_{i \in I}$ of a collection of groups is the torsion subgroup of $\Pi G_{i}$, which we denote by $\Pi^{t} G_{i}$. The $t$-product is clearly the direct product (in the homological sense) in the category of abelian $p$-groups. If for each positive integer $n, B_{n}$ is a direct sum of copies of $Z_{p^{n}}$, then $\Pi^{t} B_{n}$ is the usual way to express a torsion-complete group. In fact, in [IO] it was shown that $\prod^{t} G_{i}$ is torsion-complete iff this is true for each $G_{i}$. The $t$-product is, in certain respects, less well behaved than the usual direct product in the category of all abelian groups. For example, if $A_{i}$ is a subgroup of $G_{i}$, it will frequently be the case that the induced map $\prod^{t} G_{i} \rightarrow \prod^{t}\left(G_{i} / A_{i}\right)$ will fail to be surjective.

Let $\mathscr{R}$ be the smallest class containing the cyclic groups, which is closed with respect to (possibly infinite) direct sums and $t$-products. This class is analogous to the Reid class (the smallest class containing the infinite cyclic group, closed with respect to direct sums and direct products), which has received considerable attention by torsion-free group theorists (see, for example, [DuZ, Iv]). Let $\mathscr{R}^{s}$ be the class of groups isomorphic to summands of groups in $\mathscr{R}$. The central result of this work (Theorem 2) states that the groups in $\mathscr{R}^{s}$ are characterized by their socles. Since a direct sum of torsion-complete groups is clearly in $\mathscr{R}$, this generalizes the result of $[\mathrm{H} 1]$ mentioned above.

We will be discussing homomorphism defined on potentially large products. When this is the case, it is frequently helpful to assume that all cardinals are nonmeasurable. This use of set-theory in abelian groups goes back to [Lo]. The assumption is not overly strict, however, since measurable cardinals, if they exist at all, are extremely large. In fact, in several standard set-theoretic environments, such as the constructible universe, it can be shown that all cardinals are nonmeasurable. This assumption is necessary in that our results depend upon the following, which is a variant on a result that has appeared in various forms in [Ch, DuZ, E, Iv, K], etc.

Main Lemma. Suppose $\left\{V_{i}\right\}_{i \in I}$ and $\left\{W_{j}\right\}_{j \in J}$ are collections of valuated vector spaces with no nonzero elements of infinite value. If $\phi: \prod V_{i} \rightarrow \bigoplus W_{j}$ is a (valuated) homomorphism, then there is a positive integer $n$ and finite subsets $I_{0}, J_{0}$ of $I, J$ such that

$$
\phi\left(\prod_{I-I_{0}} V_{i}(n)\right) \subseteq \bigoplus_{J_{0}} W_{j} .
$$

Proof. The result is standard, but because of its pivotal position in this work we include an outline. If $I$ is countable and the result failed, we could inductively construct an element of the domain that would be mapped to an element of the codomain with an infinite number of nonzero coordinates, which cannot happen. The extension to the case where $I$ is nonmeasurable is then an exercise in Boolean algebras.

As was noted in $[\mathrm{K}]$, this lemma fails in the presence of measurable cardinals, so our techniques strongly depend upon the standing hypothesis of nonmeasurability.

We associate with every ordinal $\alpha$ subclasses $\mathscr{S}_{\alpha}$ and $\mathscr{T}_{\alpha}$ of $\mathscr{R}$ as follows: let $\mathscr{S}_{0}=\mathscr{T}_{0}$ be the cyclic groups. Having defined $\mathscr{S}_{\beta}$ and $\mathscr{T}_{\beta}$ for all $\beta<\alpha$, 
let $\mathscr{S}_{\alpha}$ (resp., $\mathscr{T}_{\alpha}$ ) be the groups that are isomorphic to a direct sum (resp., $t$-product) of a collection $\left\{G_{i}\right\}_{i \in I}$ where each $G_{i}$ is in $\mathscr{T}_{\beta_{i}}$ (resp., $\mathscr{S}_{\beta_{i}}$ ) for some $\beta_{i}<\alpha$. Clearly $\mathscr{S}_{\alpha}$ and $\mathscr{T}_{\alpha}$ are closed with respect to direct sums and $t$-products, respectively. Let $\mathscr{R}_{\alpha}=\mathscr{S}_{\alpha} \cup \mathscr{T}_{\alpha}$. The following summarizes some elementary properties of these classes.

Lemma 1. (a) If $\beta<\alpha$, then $\mathscr{R}_{\beta} \subseteq \mathscr{S}_{\alpha} \cap \mathscr{T}_{\alpha}$.

(b) $\mathscr{R}$ is the union of the $\mathscr{R}_{\alpha}$ over all ordinals $\alpha$.

(c) If $G$ is in any of the above classes and $n$ is a positive integer, then $G \cong$ $B \oplus X$, where $B$ is a maximal $p^{n}$-bounded subgroup of $G$ and $X$ is in the same class as $G$.

(d) All of these classes are closed with respect to finite direct sums and finite t-products.

Proof. (a) Clearly $\mathscr{S}_{\beta} \subseteq \mathscr{S}_{\alpha}$, and every element of $\mathscr{S}_{\beta}$ can be thought of as a $t$-product over a single element set, so $\mathscr{S}_{\beta} \subseteq \mathscr{T}_{\alpha}$, giving $\mathscr{S}_{\beta} \subseteq \mathscr{S}_{\alpha} \cap \mathscr{T}_{\alpha}$. Similarly, $\mathscr{T}_{\beta} \subseteq \mathscr{S}_{\alpha} \cap \mathscr{T}_{\alpha}$, and the results follows.

(b) We need to show that $\bigcup \mathscr{R}_{\alpha}$ is closed under direct sums and $t$-products. If $\left\{G_{i}\right\}_{i \in I}$ and $G_{i} \in \mathscr{R}_{\alpha_{i}}$, then by (a) each $G_{i} \in \mathscr{T}_{\alpha_{i}^{+}}$, so if $\alpha>\alpha_{i}^{+}$for each $i$, then $\bigoplus G_{i} \in \mathscr{S}_{\alpha}$, as required. The proof for $t$-products is similar.

(c) If $G_{i}=B_{i} \oplus X_{i}$, where $B_{i}$ is a maximal $p^{n}$-bounded summand of $G_{i}$, then $\bigoplus B_{i}$ (resp., $\prod^{t} B_{i}$ ) is a maximal $p^{n}$-bounded summand of $\bigoplus G_{i}=$ $\left(\bigoplus B_{i}\right) \oplus\left(\bigoplus X_{i}\right)$ (resp., $\left.\prod^{t} G_{i}=\left(\prod^{t} B_{i}\right) \oplus\left(\prod^{t} X_{i}\right)\right)$. So in inductively building up an element of one of these classes, we can separate the terms of height less than $p^{n}$ from the others, giving the result.

(d) is more or less clear, since finite direct sums and $t$-products are interchangeable.

For $G \in \mathscr{R}$, let $\mu(G)$ be the smallest $\alpha$ such that $G$ is in $\mathscr{R}_{\alpha}$.

If $G$ and $H$ are groups, we say a homomorphism $\phi: G[p] \rightarrow H[p]$ is extendable if there is a homomorphism $\theta: G \rightarrow H$ that restricts to $\phi$. If $\phi$ is extendable, it must be a homorphism in the category of valuated vector spaces. The following is the most important step in the proof of our main result.

Lemma 2. If $G$ and $H$ are in $\mathscr{R}$ and $\phi: G[p] \rightarrow H[p]$ is a homomorphism of valuated vector spaces, then $\phi$ is extendable.

Proof. We induct on $\alpha=\mu(G)$. If $\alpha=0$, then $G$ is cyclic, and clearly $\phi$ is extendable. Suppose, then, that the result is true for all groups $G^{\prime} \in \mathscr{R}$ with $\mu\left(G^{\prime}\right)<\alpha$.

Case 1. $G$ is in $\mathscr{S}_{\alpha}$. There is a collection $\left\{G_{i}\right\}_{i \in I}$ such that $G=\bigoplus G_{i}$ and for each $i, \mu\left(G_{i}\right)<\alpha$. If $\phi_{i}$ is the restriction of $\phi$ to $G_{i}$, then by induction each $\phi_{i}$ is extendable to a map $\theta_{i}$, and $\theta=\sum \theta_{i}$ is an extension of $\phi$.

Case 2. $G$ is in $\mathscr{T}_{\alpha}$. In this case, $G=\prod^{t} G_{i}$, where each $G_{i} \in \mathscr{S}_{\alpha_{i}}$ for some $\alpha_{i}<\alpha$. We induct on $\beta=\mu(H)$. If $\beta=0$, then $H$ is a cyclic group, say of order $p^{e}$. Let $G=B \oplus X$, where $B$ is a maximal $p^{e}$-bounded summand of $G$. Every element of $X[p]$ has height at least $e$, so $\phi(X[p])=0$. Since $B$ is a direct sum of cyclics, it is easily verified that $\left.\phi\right|_{B[p]}$ extends to $\theta: B \rightarrow H$, and letting $\left.\theta\right|_{X}=0$, we can conclude that $\phi$ is extendable. So assume the result is true for all groups $H^{\prime} \in \mathscr{R}$ with $\mu\left(H^{\prime}\right)<\beta$.

Subcase (i). $H$ is in $\mathscr{T}_{\beta}$. There is a collection $\left\{H_{j}\right\}_{j \in J}$ such that for each $j \in J, \mu\left(H_{j}\right)<\beta$ and $H=\prod^{t} H_{j}$. Let $\phi_{j}: G[p] \rightarrow H_{j}[p]$ be the composition 
of $\phi$ with the projection onto $H_{j}[p]$. So $\phi_{j}$ is a valuated homomorphism, and hence by induction it is extendable to $\theta_{j}: G \rightarrow H_{j}$. If $\theta: G \rightarrow H$ is the product of the $\theta_{j}$, then we see that $\phi$ is also extendable.

Subcase (ii). $H$ is in $\mathscr{S}_{\beta}$. In this case, $H=\bigoplus H_{j}$, where $H_{j} \in \mathscr{T}_{\beta_{j}}$ for some $\beta_{j}<\beta$. By Main Lemma, there are finite subsets $I_{0}, J_{0}$ of $I, J$, and a positive integer $n$ such that

$$
\phi\left(\prod_{I-I_{0}}^{t} p^{n} G_{i}[p]\right) \subseteq \bigoplus_{J_{0}} H_{j}[p] .
$$

For each $i \in I-I_{0}[p]$, let $G_{i}=B_{i} \oplus X_{i}$, where $B_{i}$ is a maximal $p^{n}$-bounded summand. Note $p^{n} G_{i}=X_{i}[p]$, and by Lemma $1(\mathrm{c})$, we may assume $X_{i} \in \mathscr{S}_{\alpha_{i}}$. Let

$$
G_{1}=\left(\prod \prod_{I_{0}}^{t} G_{i}\right) \oplus\left(\prod_{I-I_{0}}^{t} B_{i}\right) \quad \text { and } \quad G_{2}=\prod \prod_{I-I_{0}}^{t} X_{i},
$$

and so $G=G_{1} \oplus G_{2}$. Note $G_{2} \in \mathscr{T}_{\alpha}$, and by Lemma $1(\mathrm{~d}), G_{1} \in \mathscr{S}_{\alpha_{0}}$, where $\alpha_{0}=\max _{I_{0}}\left\{\alpha_{i}\right\}<\alpha$ (the second summand is bounded and hence a direct sum of cyclics). So by induction on $\alpha$, the map $\phi: G_{1}[p] \rightarrow \bigoplus_{J} H_{j}[p]$ must be extendable to a map $\theta_{1}: G_{1} \rightarrow \bigoplus_{J} H_{j}$. Again by Lemma $1(\mathrm{~d}), \bigoplus_{J_{0}} H_{j} \in \mathscr{T}_{\beta_{0}}$, where $\beta_{0}=\max _{J_{0}}\left\{\beta_{j}\right\}<\beta$. So by induction on $\beta$, the map $\phi: G_{2}[p] \rightarrow$ $\bigoplus_{J_{0}} H_{j}[p]$ extends to a map $\theta_{2}: G_{2} \rightarrow \bigoplus_{J_{0}} H_{j}$. If $\theta$ is the sum of $\theta_{1}$ and the composition of $\theta_{2}$ with the usual inclusion, then $\theta$ will extend $\phi$, as desired.

Our immediate aim is to extend this lemma to $\mathscr{R}^{s}$. We pause for the following characterization of this class.

Proposition 1. $\mathscr{R}^{s}$ is the smallest class containing the cyclic groups that is closed with respect to the formation of direct sums, summands, and t-products.

Proof. Since $\mathscr{R}^{s}$ is trivally closed with respect to summands, we need to show that it is also closed with respect to direct sums and $t$-products. But if $\left\{G_{i}\right\}_{i \in I}$ is a family of groups in $\mathscr{R}^{s}$, then each $G_{i}$ is the summand of a group $X_{i}$ in $\mathscr{R}$ and so $\oplus G_{i}$ and $\Pi^{t} G_{i}$ are summands of $\bigoplus X_{i}$ and $\Pi^{t} X_{i}$, respectively, and these latter groups are in $\mathscr{R}$.

Lemma 3. If $G$ and $H$ are in $\mathscr{R}^{s}$, then there is an $X \in \mathscr{R}$ such that $G \oplus X$ and $H \oplus X$ are in $\mathscr{R}$.

Proof. Suppose $G \oplus Y$ and $H \oplus Z$ are in $\mathscr{R}$. Consider

$$
\begin{aligned}
X & =\left(\bigoplus_{\omega} G\right) \oplus\left(\bigoplus_{\omega} Y\right) \oplus\left(\bigoplus_{\omega} H\right) \oplus\left(\bigoplus_{\omega} Z\right) \\
& \cong\left(\bigoplus_{\omega}(G \oplus Y)\right) \oplus\left(\bigoplus_{\omega}(H \oplus Z)\right) .
\end{aligned}
$$

So $G \oplus X \cong H \oplus X \cong X$ is in $\mathscr{R}$.

Theorem 1. If $G$ and $H$ are in $\mathscr{R}^{s}$ and $\phi: G[p] \rightarrow H[p]$ is a valuated vector space homomorphism, then $\phi$ is extendable.

Proof. Using Lemma 3, choose $X \in \mathscr{R}$ so that $G \oplus X$ and $H \oplus X$ are in $\mathscr{R}$. Define $\gamma:(G \oplus X)[p] \rightarrow(H \oplus X)[p]$ to be $\phi$ on the first summands and 
the identity map on the second. By Lemma $2, \gamma$ extends to a homomorphism, $\lambda: G \oplus X \rightarrow H \oplus X$. Letting $\theta: G \rightarrow H$ be the composition

$$
G \rightarrow G \oplus X \stackrel{\lambda}{\rightarrow} H \oplus X \rightarrow H,
$$

clearly $\theta$ extend $\phi$, as desired.

Lemma 4. If $\phi: G[p] \rightarrow H[p]$ extends to $\theta: G \rightarrow H$, then $\theta$ is an isomorphism iff $\phi$ is an isometry.

Proof. Sufficiency being obvious, suppose $\phi$ is an isomorphism of valuated vector spaces. If the kernel of $\theta$ was nonzero, it would have an element of order $p$. This element would also have to be in the kernel of $\phi$, which is a contradiction. So $\theta$ is injective. Consider $G^{\prime}=\theta(G)$. Since $\phi$ preserves values, it follows that $G^{\prime}$ is a pure subgroup of $H$ (see [F1, 26(h)]). But since $H[p]=\phi(G[p])=G^{\prime}[p]$, it follows that $G^{\prime}=H$ (see [F1, 26(j)]), so $\theta$ is also surjective.

Theorem 2. The groups in $\mathscr{R}^{s}$ are characterized by their socles. In fact, any isometry between the socles of groups in $\mathscr{R}^{s}$ extends to an isomorphism of the groups.

Proof. Theorem 1 and Lemma 4.

Let $\mathscr{S}_{\alpha}^{s}$ and $\mathscr{T}_{\alpha}^{s}$ be the classes of groups isomorphic to summands of $\mathscr{S}_{\alpha}$ and $\mathscr{T}_{\alpha}$, respectively. We now wish to show that for any ordinal $\alpha$, there is a $G \in \mathscr{R}$ with $\mu(G)=\alpha$. In doing so, we will actually construct two sequences of groups of increasing complexity. Note that $\mathscr{S}_{1}=\mathscr{S}_{1}^{s}$ is the direct sums of cyclics and $\mathscr{T}_{1}=\mathscr{T}_{1}^{s}$ is the torsion-completes. So $\mathscr{S}_{1} \cap \mathscr{T}_{1}$ is the bounded groups. For higher ordinals, we can construct examples using the following (cf., [DuZ, Iv]):

Theorem 3. Suppose $1<\alpha$ is an ordinal and for every $0<i<\alpha$, we have groups $G_{i} \in \mathscr{T}_{i}-\mathscr{S}_{i}^{s}$ and $H_{i} \in \mathscr{S}_{i}-\mathscr{T}_{i} s$. Then

(a) $H_{\alpha}=\bigoplus_{\omega} \bigoplus_{i<\alpha} G_{i}$ is in $\mathscr{S}_{\alpha}-\mathscr{T}_{\alpha}^{s}$,

(b) $G_{\alpha}=\prod_{\omega}^{t} \prod_{i<\alpha}^{t} H_{i}$ is in $\mathscr{T}_{\alpha}-\mathscr{S}_{\alpha}^{s}$.

Proof. Clearly $H_{\alpha} \in \mathscr{S}_{\alpha}$ and $G_{\alpha} \in \mathscr{T}_{\alpha}$.

Considering (a), suppose $H_{\alpha}$ is a summand of $\prod_{J}^{t} Y_{j}$, where each $Y_{j} \in$ $\mathscr{S}_{\alpha_{j}}$ for some $\alpha_{j}<\alpha, \pi$ is the projection onto $H_{\alpha}$, and $Z=\operatorname{ker} \pi$ is the complementary summand. By Main Lemma, there are finite sets $K, J_{0}$ of $\omega$, $J$ and a positive integer $n$ such that

$$
\pi\left(\prod_{J-J_{0}}^{t} p^{n} Y_{j}[p]\right) \subseteq \bigoplus_{K} \bigoplus_{\alpha} G_{i}[p] .
$$

Letting $M_{0}=\bigoplus_{K} \bigoplus_{\alpha} G_{i}$ and $M_{1}=\bigoplus_{\omega-K} \bigoplus_{\alpha} G_{i}$, we have

$$
\prod \prod_{J-J_{0}}^{t} p^{n} Y_{j}[p] \subseteq Z[p] \oplus M_{0}[p] .
$$

For each $j \in J$, let $B_{j}$ be a maximal $p^{n}$-bounded summand of $Y_{j}$ and $Y=$ $\prod_{J-J_{0}}^{t} B_{j} \oplus \prod_{J_{0}}^{t} Y_{j}$. Since the first term is bounded (and hence a direct sum of cyclics) and the second product is finite, there is a $\beta<\alpha$ such that $Y \in \mathscr{S}_{\beta}$. 
Note that $(*)$ implies that $M_{1}[p]$ is isometric to a summand of $Y[p]$, so $M_{1}$ is isomorphic to a summand of $Y$ by Theorem 2. But $M_{1}$ contains a summand isomorphic to $G_{\beta}$, which by assumption is not isomorphic to a summand of a group in $\mathscr{S}_{\beta}$. The resulting contradiction proves $(\mathrm{a})$.

Considering (b), suppose $G_{\alpha}$ is a summand of $\bigoplus_{J} E_{j}$, where $E_{j} \in \mathscr{T}_{\alpha_{j}}$ for some $\alpha_{j}<\alpha$. By applying Main Lemma to the inclusion map, there are finite subsets $K, J_{0}$ of $\omega, J$ and a positive integer $n$ such that

$$
\prod_{\omega-K}^{t} \prod_{\alpha}^{t} p^{n} H_{i}[p] \subseteq \bigoplus_{J_{0}} E_{j}[p] .
$$

Since $J_{0}$ is finite, there is a $\beta<\alpha$ such that $E=\bigoplus_{J_{0}} E_{j} \in \mathscr{T}_{\beta}$. If $H_{\beta}=B \oplus X$ with $B$ a maximal $p^{n}$-high subgroup, then $X[p]=p^{n} H_{\beta}[p]$ will be a (valuated) summand of $E[p]$, and hence by Theorem $2, H_{\beta}=B \oplus X$ will be isomorphic to a summand of $B \oplus E \in \mathscr{T}_{\beta}$, contrary to assumption.

Corollary 1. For every ordinal $\alpha$ there is a $G$ with $\mu(G)=\alpha$.

The groups $G_{\alpha}$ and $H_{\alpha}$ constructed above are unnecessarily large. They were chosen mostly to simplify notation. The interested reader can check that when $\alpha$ is a limit ordinal, we could let

$$
G_{\alpha}=\bigoplus_{i<\alpha} H_{i} \quad \text { and } \quad H_{\alpha}=\prod \prod_{i<\alpha}^{t} G_{i}
$$

and when $\alpha$ is isolated, we could let

$$
G_{\alpha}=\bigoplus_{\omega} H_{\alpha-1} \quad \text { and } \quad H_{\alpha}=\prod \prod_{\omega}^{t} G_{\alpha-1},
$$

and the above proof goes through without significant change.

Corollary 2. The groups $G_{\alpha}, H_{\alpha}$ constructed above are all nonisomorphic.

Proof. They clearly belong to different classes.

We conclude with a couple of questions.

(1) To what extent do the above results hold in the presence of measurable cardinals? Recall that Main Lemma is definitely not valid in this case.

(2) For an ordinal $\alpha$, does $\mathscr{S}_{\alpha}=\mathscr{S}_{\alpha}^{s}$ ? Similarly, does $\mathscr{T}_{\alpha}=\mathscr{T}_{\alpha}^{s}$ ? This is clear when $\alpha=1$. In [H2] it was shown that a summand of a group isomorphic to a direct sum of torsion-completes is also of this form. In other words, $\mathscr{S}_{2}=$ $\mathscr{S}_{s}^{2}$. In [La] It was shown that a summand of a countable $t$-product of groups that are direct sums of cyclics will once again be of this form. This is evidence that $\mathscr{T}_{2}=\mathscr{T}_{2}^{s}$ is valid, but the proof utilized in [La] strongly depends upon the countability of the $t$-product.

\section{REFERENCES}

[Ch] S. Chase, On direct sums and products of modules, Pacific J. Math. 12 (1962), 847-854.

$[\mathrm{Cu}]$ D. Cutler, Abelian p-groups determined by their $p^{n}$-socles, Abelian Group Theory, Gobel and Walker, (eds.), Gordon and Breach, New York 1986, pp. 111-116.

[Du] M. Dugas, On socles of abelian p-groups in L, Rocky Mountain J. Math. 14 (1988), 733752. 
[DuZ] M. Dugas and B. Zimmermann-Huisgen, Iterated direct sums and products of modules, Lecture Notes in Math., vol. 874, Springer-Verlag, New York, 1982.

[E] K. Eda, A boolean power and a direct product of abelian groups, Tsukuba J. Math. 6 (1982), 187-194.

[F1] L. Fuchs, Infinite Abelian groups, vols. 1, 2, Academic Press, New York, 1970, 1973.

[F2] _ Vector spaces with valuations, J. Algebra 35 (1978), 23-38.

[FI] L. Fuchs and J. Irwin, On $p^{\omega+1}$-projective p-groups, Proc. London Math. Soc. (2) 30 (1975), 459-470.

[H1] P. Hill, A classification of direct sums of closed groups, Acta Math. Acad. Sci. Hungar. 17 (1966), 263-266.

[H2] _ The isomorphic refinement theorem for direct sums of closed groups, Proc. Amer. Math. Soc. 18 (1967), 913-919.

[HM] P. Hill and C. Megibben, On primary groups with countable basic subgroups, Trans. Amer. Math. Soc. 124 (1966), 49-59.

[IO] J. Irwin and J. O'Neill, On direct products of abelian groups, Canad. J. Math. 22 (1970), 525-544.

[Iv] Ivanov, Direct sums and complete direct sums of abelian groups, Abelian Groups and Modules, (L. A. Skornyakov, ed.), Tomsk. Gos. Univ., Tomsk, 1980, pp. 70-90.

[K] P. Keef, On products of primary abelian groups, to appear, J. Algebra.

[La] L. Lady, Countable torsion products of abelian p-groups, Proc. Amer. Math. Soc. 37 (1973), $10-16$.

[Lo] J. Los, On the complete direct sum of countable abelian groups, Publ. Math. Debrecen 3 (1954), 269-272.

[MS] A. Mekler and S. Shelah, Determining abelian p-groups from their $n$-socles, Comm. in Algebra 18 (1990), 287-307.

[S] S. Shelah, On reconstructing separable reduced p-groups with a given socle, Israel J. Math. 60 (1987), 146-166.

Department of Mathematics, Whitman College, Walla Walla, Washington 99362 